\title{
Visual Presentation and Factors Affecting Visual Outcome in Children with Craniopharyngioma in East Coast States of Peninsular Malaysia: A Five-year Review
}

Ismail M. Mohd-Ilham ${ }^{1}$, Ghanimi Ramli Ahmad-Kamal ${ }^{1}$, Wan-Hazabbah Wan Hitam ${ }^{1}$, Ismail Shatriah $^{2}$

1. Ophthalmology, School of Medical Sciences, Universiti Sains Malaysia, Kubang Kerian, MYS 2. Department of Ophthalmology, School of Medical Sciences, Universiti Sains Malaysia, Kubang Kerian, MYS

$\square$ Corresponding author: Ismail Shatriah, shatriah@usm.my

Disclosures can be found in Additional Information at the end of the article

\section{Abstract}

\section{Purpose}

To describe the visual presentation and factors affecting visual outcome in pediatric patients treated for craniopharyngioma at a referral center in the East Coast states of Peninsular Malaysia.

\section{Methodology}

A retrospective review of medical records of children aged 17 years and below who had been treated for craniopharyngioma in Hospital Universiti Sains Malaysia from January 2014 to December 2018. The data collected included age, gender, presenting symptoms and duration, visual acuity, visual fields, color vision, light brightness, relative afferent pupillary defects, fundus examination and cranial nerves examination. The best corrected visual acuity during presentation, and after a one-year post-operative period, was documented. Records on investigations, surgical procedures, therapeutic modalities and recurrences were also reviewed.

\section{Results}

A total of 11 pediatric patients (22 eyes) were recruited. Fifty percent presented with optic atrophy. The mean duration of the onset of symptoms before consultation was 22.3 (24.5) months. A final best corrected visual acuity of $6 / 12$ (20/40) or better was observed in $50 \%$ of the patients. There was a statistically significant association between presenting visual acuity, optic nerve function and visual field defects, and the final visual outcome.

Received 03/26/2019

Review began 03/30/2019 Review ended 04/02/2019 Published 04/08/2019

(c) Copyright 2019

Mohd-Ilham et al. This is an open access article distributed under the terms of the Creative Commons Attribution License CC-BY 3.0., which permits unrestricted use, distribution, and reproduction in any medium, provided the original author and source are credited.

\section{Conclusions}

Visual presentations in our study were fairly similar to previous reported studies. One-third presented late with permanent visual loss. Almost half had significant visual impairment after one-year post-operative period. Significant associations were observed between presenting visual acuity, duration of symptoms, impairment of optic nerve function tests, and visual field defects during presentation, and final visual acuity at one year after treatment.

Categories: Neurology, Ophthalmology

How to cite this article

Mohd-Ilham I M, Ahmad-Kamal G, Wan Hitam W, et al. (April 08, 2019) Visual Presentation and Factors Affecting Visual Outcome in Children with Craniopharyngioma in East Coast States of Peninsular Malaysia: A Five-year Review. Cureus 11(4): e4407. DOI 10.7759/cureus.4407 
Keywords: pediatric craniopharyngioma . visual presentation . final visual outcome

\section{Introduction}

Craniopharyngioma is a rare intracranial tumor. It is a benign epithelial tumor originating from the remnants of Rathke's pouch, and it is localized in either the sellar or parasellar regions [1]. The systemic sequelae and visual prognosis are strongly related to the tumor's close proximity to surrounding vital structures. The anterior visual pathway is among the most common anatomical structure being compressed by the tumor, leading to a classical visual presentation of bitemporal hemianopia, inferior quadrantanopia and optic nerve atrophy.

The incidence rate for Western countries is estimated at 0.13 per 100,000 people annually [2]. In contrast, the annual rate for Asian countries is even higher with an estimated 0.24 to 0.53 incidents per 100,000 [3-5]. Reports on final visual outcome for patients with pediatric craniopharyngioma have been published in Europe, the Middle East and the United States [6$10]$.

Based on a PubMed search, the only data available on visual outcomes for Asian children with craniopharyngioma was from Korea [11-12]. This study aims to describe the visual presentation and factors affecting visual outcome for pediatric patients with craniopharyngioma in the East Coast states of Peninsular Malaysia, and to discuss the existing literature.

\section{Materials And Methods}

A retrospective survey was performed on pediatric patients (age 17 years old or younger) with craniopharyngioma who were treated at Hospital Universiti Sains Malaysia in Kelantan, Malaysia, from January 2014 to December 2018, and who received a follow-up for at least one year after treatment. This study was conducted in accordance with the Declaration of Helsinki, and the study protocol was approved by the Research and Ethical Committee, School of Medical Sciences, Universiti Sains Malaysia. Hospital Universiti Sains Malaysia is the leading referral center for the East Coast states of Malaysia for pediatric neurology and ophthalmology services.

All children diagnosed with craniopharyngioma, based on clinical, radiological and histopathological examination, were included in this study. Those with pre-existing ocular problems such as amblyopia, other causes of optic neuropathy and infiltrative diseases, were excluded.

The data collected included age, gender, presenting symptoms and duration, visual acuity, visual fields, color vision, light brightness, relative afferent pupillary defect, fundus examination and cranial nerves examination. The best corrected visual acuity during presentation, and after a one-year post-operative period, was documented.

Visual fields were tested using confrontational test or by using the Humphrey visual field test. Visual fields were described as bitemporal hemianopia, homonymous hemianopia, quadrantanopia, unilateral temporal field defect, scotoma or normal fields. Records on investigations, surgical procedures, therapeutic modalities and recurrences were also reviewed.

Visual acuity was classified as 'good' if the best corrected visual acuity was better than or equal to 6/12 (20/40) during presentation or at the one-year post-operative review. Data was analyzed using the Statistical Package for the Social Sciences for Windows version 24 (SPSS Inc., Chicago, IL, USA). Factors affecting the final visual outcome were further analyzed based on the Fisher exact test. 


\section{Cureus}

\section{Results}

Table 1 represents clinical profile of the study population. A total of 11 pediatric patients (22 eyes) with craniopharyngioma were recruited according to the study protocol. A majority of the patients were male (63.6\%), ranging in age from 3 to 16 years, with the mean age of $9.5(4.2)$ years. The mean duration for the onset of symptoms before consultation was 22.3 (24.5) months, ranging from one month to five years.

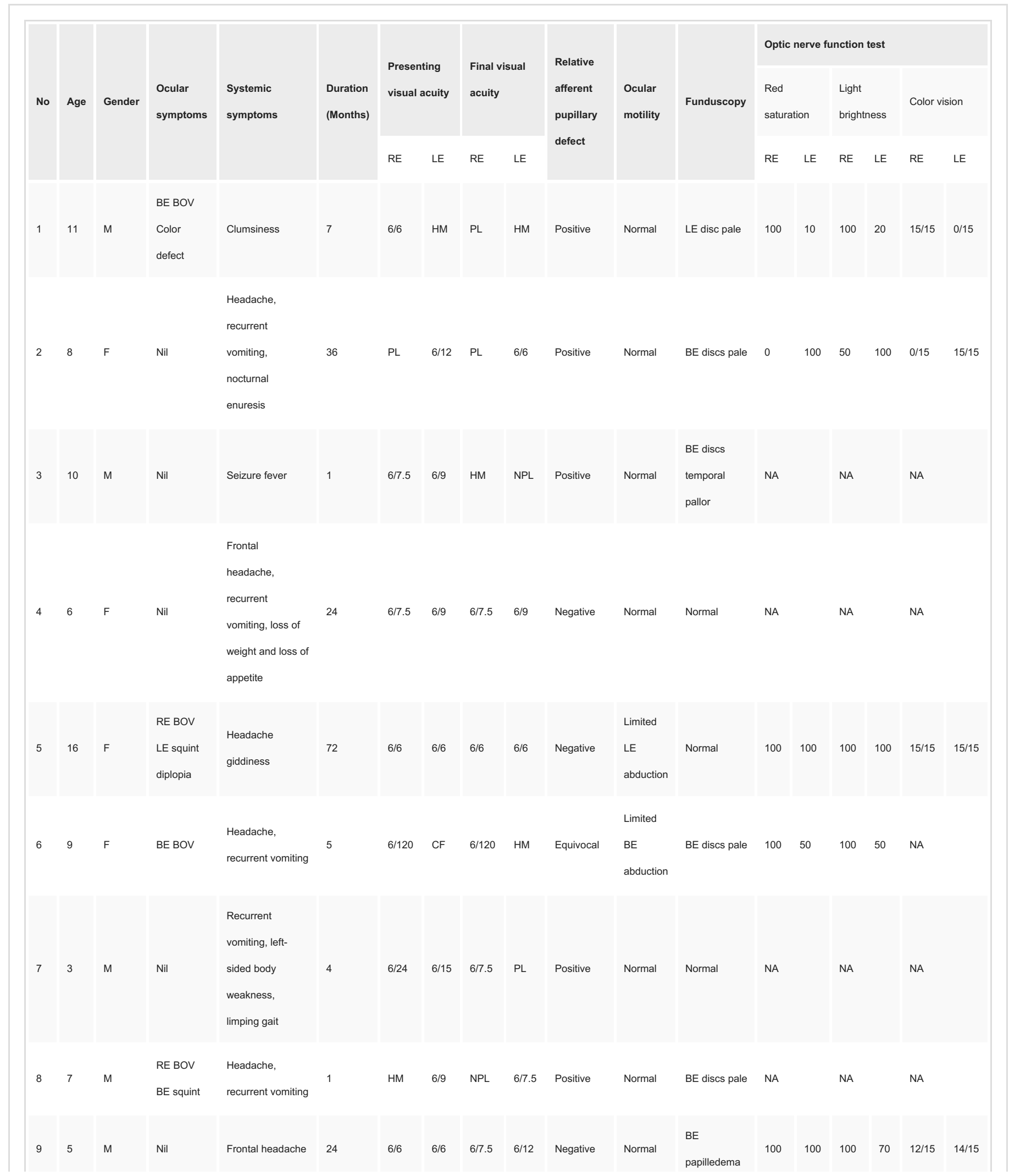




\section{Cureus}

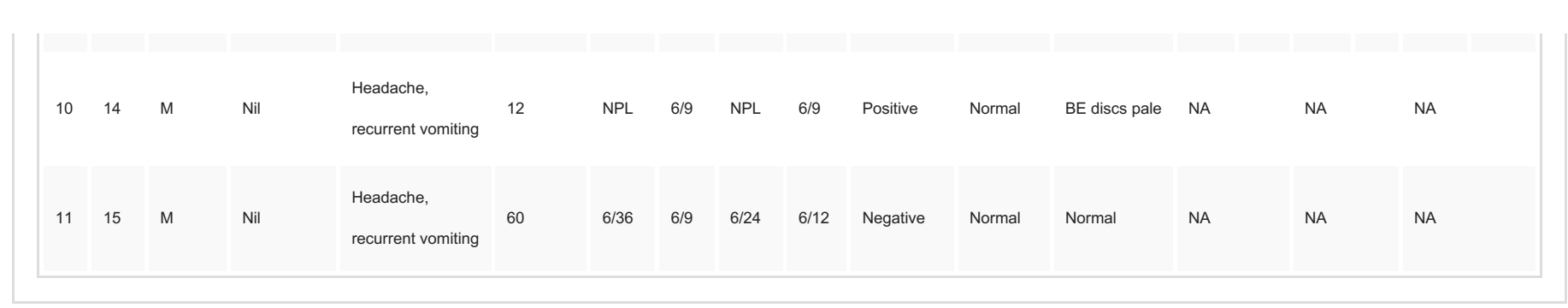

\section{TABLE 1: Clinical profile.}

M: Male; F: Female; BE: Both eyes; RE: Right eye; LE: Left eye; BOV: Blurring of vision; HM: Hand movement; PL: Perception of light; CF: Counting fingers; NPL: No perception of light; NA: Not available. Color vision based on Ishihara Color chart.

A majority of the patients $(63.6 \%, \mathrm{n}=7)$ had no visual symptoms during presentation. Four patients (36.4\%) presented with visual loss and two patients (18.2\%) showed signs of strabismus. Nine eyes (40.9\%) presented a best corrected visual acuity worse than 6/15 (20/50), while a final best corrected visual acuity of 6/12 (20/40) or better was observed in 11 patients (50.0\%). Optic atrophy was documented in 11 patients (50.0\%). Four patients (18.1\%) manifested defects in color vision, while seven patients (31.8\%) had relative afferent pupillary defects, and two patients (9.1\%) exhibited evidence of papilledema on presentation. Eleven eyes (50.0\%) had visual field defects. Out of these 11 eyes, the most common visual field defect was temporal hemianopia (22.7\%, five eyes). Other visual field defects included central scotoma (9.1\%, two eyes), inferior scotoma ( $4.6 \%$, one eye), and quadrantanopia ( $9.1 \%$, two eyes). We were unable to perform visual field testing on the remaining five eyes $(22.7 \%)$ due to lack of cooperation. These are summarized in Table 2. 


\section{Cureus}

\begin{tabular}{lc} 
Variables & No (\%) \\
\hline Total patient $(\mathrm{n})$ & 11 \\
$\begin{array}{l}\text { Age } \\
\quad \text { Mean (SD), years }\end{array}$ \\
$\quad$ Range, years & $9.5(4.2)$ \\
Gender & $3-16$ \\
$\quad$ Male & \\
$\quad$ Female & $7(63.6)$ \\
\end{tabular}

Presenting visual acuity*

$\begin{array}{ll}6 / 6-6 / 12 & 13(59.0) \\ 6 / 15-6 / 60 & 3(13.6) \\ \text { Worse than } 6 / 60 & 6(27.3)\end{array}$

Final visual acuity*

$6 / 6-6 / 12$

$11(50.0)$

$6 / 15-6 / 60$

Worse than $6 / 60$

$10(45.5)$

Visual field defects*

Hemianopia

Bilateral

$2(9.1)$

Unilateral

$3(13.6)$

Scotoma

$3(13.6)$

Quadrantanopia

$2(9.1)$

Constricted visual field

Normal

Not available

$5(22.7)$

\section{TABLE 2: Demographic and clinical data.}

${ }^{*}$ Calculated based on 22 eyes.

SD: Standard deviation 


\section{Cureus}

Table 3 shows the investigation and treatment profile of pediatric patients with craniopharyngioma. Computed tomography (CT) scans and magnetic resonance imaging (MRI) were performed on all patients. Evidence of suprasellar masses were documented for all patients. A majority of the lesions were cystic (63.6\%) and, four patients (36.4\%) displayed evidence of mixed cystic and solid lesions. Most of our patients (75\%) underwent a total resection of the mass, while one patient (12.5\%) had the tumor biopsied.

\begin{tabular}{|c|c|c|c|c|c|c|c|c|c|c|c|}
\hline \multirow{3}{*}{ No } & \multicolumn{4}{|c|}{ Visual field on presentation } & \multirow{2}{*}{\multicolumn{3}{|c|}{ Imaging (CT scan/MRI) }} & \multirow{3}{*}{ Histopathology } & \multirow{3}{*}{ Surgical intervention } & \multirow{3}{*}{$\begin{array}{l}\text { Follow- } \\
\text { up } \\
\text { (months) }\end{array}$} & \multirow{3}{*}{ Recurrence } \\
\hline & \multicolumn{2}{|c|}{ Humphrey VF } & \multicolumn{2}{|c|}{ Confrontation test } & & & & & & & \\
\hline & RE & LE & RE & LE & Location & Extension & Characteristics & & & & \\
\hline 1 & NA & Inferior scotoma & NA & & Suprasellar & $\begin{array}{l}\text { Prepontine, } \\
\text { frontal inter- } \\
\text { hemispheric } \\
\text { region }\end{array}$ & $\begin{array}{l}\text { Cystic calcified } \\
\text { lobulated }\end{array}$ & NA & Refuse intervention & 36 & NA \\
\hline 2 & NA & & $\begin{array}{l}\text { Temporal } \\
\text { hemianopia }\end{array}$ & $\begin{array}{l}\text { Temporal } \\
\text { hemianopia }\end{array}$ & Suprasellar & $\begin{array}{l}\text { Third ventricle, } \\
\text { floor of sellar } \\
\text { turcica }\end{array}$ & $\begin{array}{l}\text { Cystic } \\
\text { lobulated }\end{array}$ & Adamantinomatous & $\begin{array}{l}\text { Bifrontal craniotomy } \\
\text { and tumor excision }\end{array}$ & 40 & Yes \\
\hline 3 & $\begin{array}{l}\text { Supero- } \\
\text { temporal } \\
\text { quadrant- } \\
\text { anopia }\end{array}$ & $\begin{array}{l}\text { Temporal } \\
\text { hemianopia }\end{array}$ & NA & & Suprasellar & Cavernous sinus & $\begin{array}{l}\text { Mixed solid } \\
\text { and cystic }\end{array}$ & Adamantinomatous & $\begin{array}{l}\text { craniotomy with tumor } \\
\text { debulking }\end{array}$ & 16 & Yes \\
\hline 4 & Normal & Normal & Normal & Normal & Suprasellar & Nil & $\begin{array}{l}\text { Mixed solid } \\
\text { and cystic }\end{array}$ & Adamantinomatous & $\begin{array}{l}\text { Vertex craniotomy with } \\
\text { tumor debulking }\end{array}$ & 8 & No \\
\hline 5 & Normal & Normal & Normal & Normal & Suprasellar & Anterior pons & $\begin{array}{l}\text { Cystic calcified } \\
\text { multi-lobulated }\end{array}$ & NA & Refuse intervention & 17 & NA \\
\hline 6 & NA & & $\begin{array}{l}\text { Central } \\
\text { scotoma }\end{array}$ & $\begin{array}{l}\text { Central } \\
\text { scotoma }\end{array}$ & Suprasellar & Third ventricle & Cystic & Adamantinomatous & $\begin{array}{l}\text { Ventriculoperitoneal } \\
\text { shunt with tumor } \\
\text { debulking }\end{array}$ & 20 & Yes \\
\hline 7 & NA & & NA & & Suprasellar & $\begin{array}{l}\text { Anterior } \\
\text { commissural, } \\
\text { Midbrain, Pons }\end{array}$ & $\begin{array}{l}\text { Cystic calcified } \\
\text { lobulated }\end{array}$ & Adamantinomatous & $\begin{array}{l}\text { Left pterional } \\
\text { craniotomy with tumor } \\
\text { debulking }\end{array}$ & 24 & Yes \\
\hline 8 & NA & & NA & $\begin{array}{l}\text { Temporal } \\
\text { hemianopia }\end{array}$ & Suprasellar & Nil & Cystic calcified & Adamantinomatous & $\begin{array}{l}\text { Right pterional } \\
\text { craniotomy with tumor } \\
\text { debulking }\end{array}$ & 24 & Yes \\
\hline 9 & NA & & Normal & Normal & Suprasellar & $\begin{array}{l}\text { Hypothalamic } \\
\text { region }\end{array}$ & $\begin{array}{l}\text { Mixed solid } \\
\text { and cystic } \\
\text { lobulated }\end{array}$ & Adamantinomatous & $\begin{array}{l}\text { Ventriculoperitoneal } \\
\text { shunt with endoscopic } \\
\text { tumor biopsy }\end{array}$ & 36 & No \\
\hline 10 & NA & & NA & $\begin{array}{l}\text { Temporal } \\
\text { hemianopia }\end{array}$ & Suprasellar & $\begin{array}{l}\text { Optic chiasm, } \\
\text { pituitary fossa }\end{array}$ & $\begin{array}{l}\text { Mixed solid } \\
\text { and cystic }\end{array}$ & NA & $\begin{array}{l}\text { Right supraorbital } \\
\text { keyhole craniotomy } \\
\text { with tumor debulking }\end{array}$ & 11 & No \\
\hline 11 & Constricted & $\begin{array}{l}\text { Inferior- } \\
\text { temporal }\end{array}$ & NA & NA & Suprasellar & $\begin{array}{l}\text { Third ventricle, } \\
\text { anterior part of }\end{array}$ & Cystic calcified & NA & $\begin{array}{l}\text { Left frontal burr hole } \\
\text { with endoscopic }\end{array}$ & 48 & Yes \\
\hline
\end{tabular}




\section{Cureus}

\section{TABLE 3: Investigations and treatment profile.}

NA: Not available; CT: Computed tomography; MRI: Magnetic resonance imaging; VF: Visual field; LE: Left eye; RE: Right eye.

In Table 4, we analyzed the associations between age, gender, best corrected visual acuity during presentation, duration of symptoms before treatment, impaired optic nerve function tests, visual field defects, tumor size, type of surgery and recurrence with good visual acuities after the one-year post-operative period. The analysis produced evidence of significant associations between best corrected visual acuity during presentation, duration of symptom prior to consultation, impaired optic nerve function tests, visual field defects and good visual acuity after one-year post-operative period ( $\mathrm{P}<0.05$, Fisher exact test).

\section{Variables}

Age of diagnosis (years)

Less than six years old

Six years old and older

Gender

Male

Female

VA presentation*

$6 / 6-6 / 12$

Worse than $6 / 15$

Duration of symptoms prior to consultation

Less than 12 months

12 months and longer

Optic nerve function*

Impaired

Normal

Visual field*

Impaired

Normal

Imaging findings

\section{Frequency, $\mathrm{n}(\%) \quad$ Final visual acuity (6/12 or Better), n (\%) p-value}

0.074

$\begin{array}{ll}6(27.3) & 5(22.7) \\ 16(72.7) & 6(27.3)\end{array}$

$16(72.7)$

$14(63.6)$

$6(27.3)$

$8(36.4)$

$5(22.7)$

$9(40.9)$ 


\section{Cureus}

Tumor size (by greatest dimension)

$\begin{array}{lcc}4 \mathrm{~cm} \text { and greater } & 12(54.5) & 9(69.2) \\ \text { Less than } 4 \mathrm{~cm} & 10(45.5) & 4(30.8)\end{array}$

Treatment

Total resection

$16(72.7)$

$8(39.4)$

Partial resection/biopsy

$2(9.1)$

Refused surgery

$4(18.2)$

Recurrence

Yes

$6(54.5)$

$4(18.2)$

No

$3(27.3)$

$5(22.7)$

Missing

$2(18.2)$

$2(9.1)$

\section{TABLE 4: Factors affecting good final visual acuity.}

*Based on 22 eyes; $p$-value $<0.05$ is statistically significant, Fisher Exact Test.

VA: Visual acuity

\section{Discussion}

Improved visual outcome is the main concern for ophthalmologists and neurosurgeons managing pediatric craniopharyngioma. Table 5 summarizes published reports on visual presentation and outcome in patients with pediatric craniopharyngioma from Asian countries, the United Kingdom, Spain, Israel, and France, as well as those included in our study [6, 8-13].

\begin{tabular}{|c|c|c|c|c|c|c|c|}
\hline Author (Year) & $\begin{array}{l}\text { Our } \\
\text { study } \\
\text { (2019) }\end{array}$ & $\begin{array}{l}\text { Drimtzias et } \\
\text { al. (2014) [6] }\end{array}$ & $\begin{array}{l}\text { Puget et al. } \\
\text { (2007) [13] }\end{array}$ & $\begin{array}{l}\text { Mediero et } \\
\text { al. (2015) [8] }\end{array}$ & $\begin{array}{l}\text { Lee et al. } \\
\text { (2012) [11] }\end{array}$ & $\begin{array}{l}\text { Jung et al. } \\
(2010) \text { [12] }\end{array}$ & $\begin{array}{l}\text { Bialer et al. } \\
\text { (2012) [9] }\end{array}$ \\
\hline Country & Malaysia & $\begin{array}{l}\text { United } \\
\text { Kingdom }\end{array}$ & France & Spain & Korea & Korea & Israel \\
\hline \multicolumn{8}{|l|}{ Population } \\
\hline Adult & 0 & 0 & 0 & 0 & 0 & 0 & 0 \\
\hline Children & 11 & 20 & 66 & 10 & 27 & 17 & 20 \\
\hline Mean age (SD) & $9.5(4.2)$ & 7.3 & 7.4 & 5 & U & 12 & $6.5(3.9)$ \\
\hline \multicolumn{8}{|l|}{ Gender, n (\%) } \\
\hline Male & 7 (63.6) & $10(50.0)$ & 42 (63.6) & $6(60.0)$ & 15 (55.6) & 12 (70.6) & $10(50.0)$ \\
\hline
\end{tabular}


Cureus

$\begin{array}{llllllll}\text { Female } & 4(36.4) & 10(50.0) & 24(36.4) & 4(40.0) & 12(44.4) & 5(16.7) & 10(50.0) \\ \begin{array}{l}\text { Systemic } \\ \text { symptoms, n (\%) }\end{array} & & & & & & & \\ \text { Headache } & 8(72.7) & 14(70.0) & \mathrm{U} & \mathrm{U} & 18(66.7) & 13(76.5) & 6(55.0) \\ \text { Nausea/vomiting } & 7(63.6) & \mathrm{U} & \mathrm{U} & \mathrm{U} & 6(22.2) & \mathrm{NS} & \mathrm{U} \\ \text { Motor deficit } & 2(18.2) & \mathrm{U} & 8(12.0) & \mathrm{U} & \mathrm{U} & 0(0) & \mathrm{U} \\ \text { Seizure } & 1(9.1) & \mathrm{U} & 3(4.5) & \mathrm{U} & \mathrm{U} & 0(0) & 1(9.0)\end{array}$

Ocular symptoms,

n (\%)

$\begin{array}{llllllll}\text { Visual loss } & 4(36.4) & 6(30.0) & 29(44.0) & U & 12(44.4) & 4(23.5) & 1(9.0) \\ \text { Color vision defect } & 1(9.1) & U & U & U & U & 0(0) & U \\ \text { Diplopia/strabismus } & 2(18.2) & U & U & U & 4(14.8) & 0(0) & 3(27.0)\end{array}$

VA presentation ${ }^{*}, \mathrm{n}$

(\%)

\begin{tabular}{|c|c|c|c|c|c|c|c|}
\hline 6/6-6/12 & $13(59.0)$ & $19(47.5)$ & $U$ & $u$ & 33 (61.1) & $U$ & $U$ \\
\hline 6/15-6/60 & $3(13.6)$ & $8(20)$ & $U$ & U & $14(25.9)$ & $U$ & U \\
\hline Worse than $6 / 60$ & $6(27.3)$ & $13(32.5)$ & $\mathrm{U}$ & U & $7(13.0)$ & U & U \\
\hline \multicolumn{8}{|l|}{ VA final ${ }^{*}, \mathrm{n}(\%)$} \\
\hline $6 / 6-6 / 12$ & $11(50.0)$ & $23(57.5)$ & U & $12(60)$ & $37(68.5)$ & $\mathrm{U}$ & U \\
\hline $6 / 15-6 / 60$ & $1(4.5)$ & $4(10.0)$ & U & $3(15.0)$ & $13(24.1)$ & $\mathrm{U}$ & U \\
\hline Worse than $6 / 60$ & $10(45.5)$ & $13(32.5)$ & U & $5(25.0)$ & $4(7.4)$ & U & U \\
\hline \multicolumn{8}{|c|}{$\begin{array}{l}\text { Fundus } \\
\text { examination*, n (\%) }\end{array}$} \\
\hline Optic atrophy & $11(50.0)$ & $12(60.0)$ & U & $15(75.0)$ & $14(30.4)$ & U & $17(42.5)$ \\
\hline Papilledema & $2(9.1)$ & $6(30.0)$ & U & $0(0)$ & $10(21.7)$ & $\mathrm{U}$ & $6(15.0)$ \\
\hline Normal finding & $8(36.4)$ & U & U & $5(25.0)$ & $22(47.8)$ & $U$ & U \\
\hline \multicolumn{8}{|c|}{ Visual field defect*, n (\%) } \\
\hline $\begin{array}{l}\text { Bilateral } \\
\text { hemianopia }\end{array}$ & $2(9.1)$ & $5(35.7)$ & $10(15.0)$ & $2(20.0)$ & $3(11.1)$ & $U$ & $4(26.7)$ \\
\hline $\begin{array}{l}\text { Unilateral } \\
\text { hemianopia }\end{array}$ & $3(13.6)$ & U & $9(14.0)$ & $2(20.0)$ & $3(11.1)$ & $U$ & $3(20.0)$ \\
\hline Central scotoma & $3(13.6)$ & U & U & $0(0)$ & $2(7.4)$ & U & $0(0)$ \\
\hline Quadrantanopia & $2(9.1)$ & U & $6(9.0)$ & $0(0)$ & $0(0)$ & $U$ & $1(6.7)$ \\
\hline
\end{tabular}




\section{Cureus}

$\begin{array}{llllllll}\text { Others } & 1(4.6) & U & 11(17.0) & 2(20.0) & 6(22.2) & U & 1(6.7) \\ \begin{array}{l}\text { Not } \\ \text { performed/available }\end{array} & 5(22.7) & U & 13(20.0) & 3(30.0) & 0(0) & U & 0(0) \\ \text { Normal visual field } & 6(27.3) & 4(28.5) & 23(35.0) & 1(10.0) & 13(48.1) & U & 6(40.0)\end{array}$

Imaging findings, $\mathrm{n}$

$(\%)$

\begin{tabular}{|c|c|c|c|c|c|c|c|}
\hline Suprasellar & $\begin{array}{l}11 \\
(100.0)\end{array}$ & $20(100.0)$ & U & U & $22(100.0)$ & $10(58.8)$ & $U$ \\
\hline $\begin{array}{l}\text { Suprasellar + } \\
\text { Intrasellar }\end{array}$ & $0(0.0)$ & $0(0)$ & U & $\mathrm{U}$ & $0(0)$ & $7(41.2)$ & $U$ \\
\hline Cystic & 7 (63.6) & $10(50.0)$ & $6(10.0)$ & $U$ & $0(0)$ & $11(64.7)$ & $U$ \\
\hline Cystic and solid & $4(36.4)$ & $10(50.0)$ & $U$ & $U$ & $22(100.0)$ & $6(35.3)$ & $U$ \\
\hline Solid & $0(0.0)$ & $0(0)$ & U & $U$ & $0(0)$ & $0(0)$ & $U$ \\
\hline Calcification & $5(45.5)$ & $12(60.0)$ & $U$ & $U$ & $18(81.8)$ & $15(88.2)$ & $U$ \\
\hline Hydrocephalus & $U$ & $U$ & $35(53.0)$ & U & $3(13.6)$ & U & U \\
\hline \multicolumn{8}{|l|}{ Treatment, n (\%) } \\
\hline Total resection & $8(72.7)$ & $7(35.0)$ & $33(50.0)$ & $10(100.0)$ & $27(100.0)$ & 12 (70.6) & NS \\
\hline $\begin{array}{l}\text { Partial } \\
\text { resection/biopsy }\end{array}$ & $1(9.1)$ & $12(60.0)$ & $33(50.0)$ & $0(0)$ & $0(0)$ & $5(29.4)$ & NS \\
\hline No surgery & $2(18.2)$ & $1(5.0)$ & $0(0)$ & $0(0)$ & $0(0)$ & $0(0)$ & NS \\
\hline \multicolumn{8}{|l|}{$\begin{array}{l}\text { Histopathology, } \mathrm{n} \\
\text { (\%) }\end{array}$} \\
\hline Squamous & $0(0)$ & $U$ & $U$ & $U$ & $U$ & $0(0)$ & $U$ \\
\hline Papillary & $0(0)$ & $U$ & $U$ & $U$ & $U$ & $1(5.9)$ & $U$ \\
\hline Adamantinoma & 7 (63.6) & $U$ & 65 (98.5) & $U$ & $U$ & $16(94.1)$ & $U$ \\
\hline Recurrence & $6(54.5)$ & $11(55.0)$ & $35(53.0)$ & $2(20.0)$ & $11(40.7)$ & $2(11.8)$ & $U$ \\
\hline
\end{tabular}

\section{TABLE 5: Comparison of published studies on pediatric craniopharyngioma.}

${ }^{*}$ Calculated based on 22 eyes in our study.

VA: Visual acuity; U: Unavailable; NS: Not specified.

The mean age of presentation was 9.5 (4.2) years in our study. In the majority of the studies reviewed, the mean age of presentation was less than 10 years [6,8,9,11-13]. In contrast, Jung et al. reported the mean age was 12 years [12]. There was a male preponderance in our study population, which is also consistent with other pediatric craniopharyngioma studies [8,11-13]. 
In contrast, Drimtzias et al. and Bialer et al. from the United Kingdom and Israel respectively, reported an equal ratio of males and females $[6,9]$.

The most common symptom reported by our patients was headache (72.7\%), which is consistent with findings documented by other studies (ranging from 55 to $76.5 \%$ ) [6,9,10-11]. However, visual loss or blurring of vision was only noted in one-third of our patients (36.4\%, four eyes). This observation is also similar to studies from the United Kingdom (30.0\%) and Korea (23.5\%) [6,12]. A slightly higher percentage was reported from a second study from Korea (44.4\%) and France (44.0\%) [11,13]. This observation suggests that these children were not able to express or may not have been aware of visual symptoms during the early stages of the disease.

Among our patients, papilledema during presentation was only observed in two eyes (9.1\%), while optic atrophy was documented in 11 eyes (50\%). This finding is also consistent with studies reported by Drimtzias et al. (30\% papilledema, $60 \%$ optic atrophy), Mediero et al. (none had papilledema, $75 \%$ showed optic atrophy) and Bialer et al. (15\% papilledema, $42.5 \%$ optic atrophy) [6,8-9]. These data indicate that the diagnosis was only confirmed at a later stage, after the disease had progressed.

We found a significant association between presenting best corrected visual acuity and good final visual outcome $(\mathrm{p}=0.040)$. Nine patients $(40.9 \%)$ presented with visual acuity worse than or equal to 6/15 (20/50) and, of this group, only two patients (9.1\%) documented good final visual acuity (6/12 or 20/40, one year after treatment). A similar pattern was reported by Repka et al. [10]. They evaluated preoperative and postoperative visual acuities in 12 children younger than 18 years with craniopharyngioma. They reported that $17 \%$ of their patients had visual acuity worse than 20/60 (6/12) in the better eye at the time of diagnosis, and $27 \%$ of their patients continued to have visual acuity worse than $20 / 40(6 / 12)$ in the better eye postoperatively [13]. In contrast, Drimtzias et al. and Lee and Hwang reported a higher percentage of visual acuity at $20 / 40(6 / 12)$ after one year $(57.5 \%, 68.5 \%$ respectively) $[6,11]$.

We observed a significant association between the duration of systemic symptoms before consultation and good final visual acuity at the one-year post-operative period $(p=0.12)$. Fourteen patients (63.6\%) presented less than one year after the onset of systemic symptoms. Four patients (18.2\%) had good final visual acuity after one year. We had difficulty finding published data on this issue for comparison.

However, Chen et al. reported a median duration of systemic symptoms of seven months in their patients [14]. Lee and Hwang documented duration of 5.2 \pm 6.8 months in children with craniopharyngioma with normal visual field, and $8.9 \pm 10.6$ months in those with abnormal visual fields [11]. However, no further analysis regarding the final visual outcome was performed by these authors $[11,14]$.

All patients were examined for optic nerve functions during their first consultation, and at each follow-up visit to detect signs of optic neuropathy. The examination included visual acuity tests, afferent pupillary defect assessment, color vision tests, light brightness and visual field assessments. Impairment of the optic nerve functions identified during diagnosis of craniopharyngioma is significantly associated with final visual acuity after treatment $(\mathrm{p}=$ 0.040). Nine patients (41\%) showed evidence of optic neuropathy, while 13 patients (59\%) showed normal optic nerve function during diagnosis. Only two patients (9.1\%) who had impaired optic nerve functions displayed good visual acuity a year after treatment.

We also noted a significant association between visual field defects and final visual acuity $(\mathrm{p}=$ 0.012). Fourteen eyes (63.6\%) had visual field defects in our series. Only four patients (18.2\%) 
had good visual acuity one year after treatment. Our findings agree with Lee and Hwang who reported that patients with normal visual fields at presentation were more likely to have better visual acuity postoperatively [11].

We found no significant association between the size of the tumor and good visual acuity post operation ( $\mathrm{p}=0.110$ ). Among our patients, $54.5 \%$ had tumor that were larger than $4 \mathrm{~cm}$, as measured on CT scans or MRI findings, based on the greatest dimension. Our findings were similar to Wan et al. who also reported 54\% of their patients had tumor sizes greater than $4 \mathrm{~cm}$. Interestingly, Wan et al. also found no significant association between tumor size and visual decline [15]. Lee and Hwang supported these findings, noting that there was no significant difference in tumor size with visual field defects [11].

We observed that six patients (54.5\%) had tumor recurrences, which is consistent with other studies that reported a recurrence rate of $40.7 \%$ to $55 \%[6,11,13]$. However, there was no significant association observed between recurrence of tumors and final visual acuity.

\section{Conclusions}

Visual presentations in our study were fairly similar to previous reported studies. One-third of our patients presented late with permanent visual loss, and almost half of our patients had significant visual impairment after one-year post-operative period. There were significant associations between presenting visual acuity, duration of symptoms, impairment of optic nerve function tests, and visual field defects during presentation, and final visual acuity at one year after treatment.

\section{Additional Information \\ Disclosures}

Human subjects: Consent was obtained by all participants in this study. Animal subjects: All authors have confirmed that this study did not involve animal subjects or tissue. Conflicts of interest: In compliance with the ICMJE uniform disclosure form, all authors declare the following: Payment/services info: All authors have declared that no financial support was received from any organization for the submitted work. Financial relationships: All authors have declared that they have no financial relationships at present or within the previous three years with any organizations that might have an interest in the submitted work. Other relationships: All authors have declared that there are no other relationships or activities that could appear to have influenced the submitted work.

\section{References}

1. Karavitaki N, Cudlip S, Adams CB, Wass JA: Craniopharyngiomas. Endocr Rev. 2006, 27:371397. 10.1210/er.2006-0002

2. Bunin GR, Surawicz TS, Witman PA, Preston-Martin S, Davis F, Bruner JM: The descriptive epidemiology of craniopharyngioma. J Neurosurg. 1998, 89:547-551.

10.3171/jns.1998.89.4.0547

3. Makino K, Nakamura H, Yano S, Kuratsu J: Population-based epidemiological study of primary intracranial tumors in childhood. Childs Nerv Syst. 2010, 26:1029-1034.

10.1007/s00381-010-1126-X

4. Dho YS, Jung KW, Ha J, Seo Y, Park CK, Won YJ, Yoo H: An updated nationwide epidemiology of primary brain tumors in Republic of Korea, 2013. Brain Tumor Res Treat. 2017, 5:16-23. 10.14791/btrt.2017.5.1.16

5. Kuratsu J, Ushio Y: Epidemiological study of primary intracranial tumors in childhood. A population-based survey in Kumamoto Prefecture, Japan. Pediatr Neurosurg. 1996, 25:240247. 10.1159/000121132

6. Drimtzias E, Falzon K, Picton S, Jeeva I, Guy D, Nelson O, Simmons I: The ophthalmic natural 
history of paediatric craniopharyngioma: a long-term review. J Neurooncol. 2014, 120:651656. 10.1007/s11060-014-1600-5

7. Jacobsen MF, Thomsen ASS, Bach-Holm D, et al.: Predictors of visual outcome in patients operated for craniopharyngioma - a Danish national study. Acta Ophthalmol. 2018, 96:39-45. 10.1111/aos. 13483

8. Mediero S, Noval S, Bravo-Ljubetic L, Contreras I, Carceller F: Visual outcomes, visual fields and optical coherence tomography in paediatric craniopharyngioma. Neuroophthalmology. 2015, 39:132-139. 10.3109/01658107.2015.1039549

9. Bialer OY, Goldenberg-Cohen N, Toledano H, Snir M, Michowiz S: Retinal NFL thinning on OCT correlates with visual field loss in pediatric craniopharyngioma. Can J Ophthalmol. 2013, 48:494-499. 10.1016/j.jcjo.2013.05.001

10. Repka MX, Miller NR, Miller M: Visual outcome after surgical removal of craniopharyngiomas . Ophthalmology. 1989, 96:195-199.

11. Lee MJ, Hwang JM: Initial visual field as a predictor of recurrence and postoperative visual outcome in children with craniopharyngioma. J Pediatr Ophthalmol Strabismus. 2012, 49:3842. 10.3928/01913913-20110208-03

12. Jung TY, Jung S, Moon KS, Kim IY, Kang SS, Kim JH: Endocrinological outcomes of pediatric craniopharyngiomas with anatomical pituitary stalk preservation: preliminary study. Pediatr Neurosurg. 2010, 46:205-212. 10.1159/000318426

13. Puget S, Garnett M, Wray A, et al.: Pediatric craniopharyngiomas: classification and treatment according to the degree of hypothalamic involvement. J Neurosurg. 2007, 106:3-12. 10.3171/ped.2007.106.1.3

14. Chen C, Okera S, Davies PE, Selva D, Crompton JL: Craniopharyngioma: a review of long-term visual outcome. Clin Exp Ophthalmol. 2003, 31:220-228. 10.1046/j.1442-9071.2003.00648.x

15. Wan MJ, Zapotocky M, Bouffet E, Bartels U, Kulkarni AV, Drake JM: Long-term visual outcomes of craniopharyngioma in children. J Neurooncol. 2018, 137:645-651. 10.1007/s11060-018-2762-3 